\title{
Complex Event Processing Using Iot Devices BASED ON ARDUINO
}

\author{
Mahir Kaya ${ }^{1}$ and Yasemin Çetin-Kaya ${ }^{2}$ \\ ${ }^{1}$ Department of Computer Engineering, Gaziosmanpaşa University, Tokat, Turkey \\ ${ }^{2}$ Department of Computer Engineering, Gaziosmanpaşa University, Tokat, Turkey
}

\begin{abstract}
Complex event processing systems have gained importance since recent developments in communication and integrated circuits technologies. Developers can easily develop many smart space systems by connecting various sensors to an Arduino as an internet of thing device. These systems are useful for many places such as factories, greenhouses (plant house) and smart-homes. Especially in plant houses when the desired humidity, temperature, light and soil moisture drops the certain level, the users should be notified through their smartphones. The sensor information is sent to a central server over the internet via an access point. The collected sensor data needs to be processed online to check whether an event is occurred or not. The event processing system based on a complex event processing tool is created on the central server. It is also an important issue to inform mobile users whenever an event occurs. A publish-subscribe event based system is implemented on the central server. A mobile user is subscribed to the desired event topic. When an event occurred, which is related with a specific topic, an alarm notification is sent to the mobile users about the event information so as to take necessary precautions.
\end{abstract}

\section{KEYWORDS}

Complex event processing, Publish-subscribe, Arduino, Event based framework.

\section{INTRODUCTION}

Today various computing nodes such as sensors and actuators are embedded into the physical world in order to help people to take necessary actions immediately and also automate daily life activities of people. A Complex Event Processing (CEP) mechanism is designed for deducing information based on sensors' data. The sensors such as light, temperature, humidity, sound and motion, soil moisture, rain are equipped to an Arduino board and the collected data is processed by a CEP engine to give the proper information to mobile users who are registered to the desired event topics.

Many researchers have tried to develop an efficient smart space system especially in home environment. These systems require information either to take some necessary action on case of an event or to help people for taking optimum decisions. Smart home is defined as "the integration of technology and services through home networking for a better quality of living"[1]. Advantages of the Smart home systems can be summarized as below [1].

- Facilitates the life.

- Saves energy and efficiency.

- Supports the elderly and disabled people.

Also, context awareness, interoperability, service detection, trust, security, privacy and transparency are the main difficulties that smart home developers meet [2]. In addition, the 
internet has evolved from connecting two computers together to Internet of Things by connecting objects surrounding us to the internet. [3,4]. The next step in the IoT is to connect objects to each other and communicate through internet [3].

Studies on wireless sensor networks enable the spread of smart spaces through the data gathering and analysis from an environment. Zoumboulakis and Roussos [5] used a wireless sensor network and aimed to extract complex events. This system consists of three steps. First, the environment data is learned by the system. Second information is determined and then inferences are made according to the collected information. Yao et al. [6] conducted a study that is carried out in the hospital environment. In this study, the RFID information was transferred over wireless sensor network to monitor and identify the patients. Also, RFID based patient health monitoring and emergency response were implemented.

Aware Home Project [7] was carried out by interdisciplinary groups of researches in the Georgia Institute of Technology. This project is aimed to improve the elderly people's quality of life and enhance their life in familiar environments. They considered designing a home model that consists of a living room, a kitchen, a laundry room, a dining room, an office, two bathrooms and two bedrooms. Because of long construction time, a prototype room was made to test the systems, which are human position tracking by means of ultrasonic sensors and recognition through floor sensors (in Table 1).

Table 1. Hardware and methods of the Aware Home project

\begin{tabular}{|l|r|l|}
\hline Aware Home Project & \\
\hline Hardware & $\bullet$ Ultrasonic sensors \\
& $\bullet$ RF technology and video, \\
& $\bullet$ Floor sensors \\
\hline Method & $\bullet$ Pattern recognition, \\
& $\bullet$ Artificial vision techniques \\
& $\bullet$ Hidden Markov models \\
\hline
\end{tabular}

Finding Frequently Lost Objects (FLOs): In order to find frequently lost objects which are keys, wallets and remote control, a system including tracking and sensing technology is implemented in Aware Home. The long range indoor positioning system was used to track the objects. The communication between user and system accomplished with LCD touch panels. Audio cues were used for guiding the user to find the lost object.

The Gator Tech Smart House project [8] was carried out by the Mobile and Pervasive Computing Laboratory of University of Florida. They worked together with the College of Public Health and Health Professions during the development of the pervasive space for the elderly and disabled people. The project is aimed to figure a supportive home that is able to detect its essential parts and users, and accomplish mapping among real world and remote monitoring services (Table 2). 
International Journal on Cloud Computing: Services and Architecture (IJCCSA) Vol. 7, No. 6, December 2017

Table 2. Function and used technology of the system [8]

\begin{tabular}{|c|c|c|}
\hline System & Function & Technology \\
\hline Smart Mailbox & Alerts the occupant when mail arrived. & ns (not specified) \\
\hline Smart Front Door & $\begin{array}{l}\text { Provides keyless entrance } \\
\text { communication with visitors }\end{array}$ & $\begin{array}{l}\text { RFID tag, microphone, } \\
\text { camera, text LCD, electric } \\
\text { latch, speakers, automatic } \\
\text { door opener. }\end{array}$ \\
\hline Driving Simulator & Assess driving ability of elderly. & Ns \\
\hline $\begin{array}{l}\text { Ultrasonic Location } \\
\text { Tracking }\end{array}$ & $\begin{array}{l}\begin{array}{l}\text { Senses movement, location } \\
\text { orientation. }\end{array} \\
\text { and }\end{array}$ & Sensor \\
\hline Smart Bathroom & $\begin{array}{l}\text { Adjusts water temperature, notify soap } \\
\text { restock }\end{array}$ & $\begin{array}{l}\text { Sensor, flush detector, } \\
\text { heating thermostats }\end{array}$ \\
\hline Smart Plug & $\begin{array}{l}\text { Associate detected electrical device and } \\
\text { lamp to remote monitoring and } \\
\text { intervention application }\end{array}$ & $\begin{array}{l}\text { Sensor, RFID reader, RFID } \\
\text { tag }\end{array}$ \\
\hline Smart Blinds & Automate the adjustment of the blinds & Remote device \\
\hline Smart Mirror & Shows vital message and reminders. & Ns \\
\hline Smart Display & $\begin{array}{l}\text { Leisure media and information follow } \\
\text { occupant from room to room }\end{array}$ & Ns \\
\hline Smart Floor & Identifies and tracks occupants & Sensor \\
\hline Smart ware & $\begin{array}{l}\text { Regulates time and power setting of the } \\
\text { frozen food and show how to cook to the } \\
\text { user. }\end{array}$ & Ns \\
\hline
\end{tabular}

According to the project group, the key constraint of the previous works in pervasive domain is that "lack of the system's ability to advance as new technologies or as an application domain matures". In order to overcome this limitation, a programmable pervasive space in that smart space seems as a runtime environment and a software library together was developed.

Hussain et al. [9] developed an RFID-based smart home application that involves a research of the elderly people in need of care. In this research, it is assumed that some tasks such as, patient care monitoring, home lighting and personalized music broadcasting with wireless sensors are automatically performed. Moreover, Killeen et al. [10] developed a wireless network application that includes both software and hardware components. In this study, data transmission and data inquiry via sensor nodes is possible. Each node consists of a power module, a wireless communication module, a processing module and a sensor/actuator module. Through inter-node data transmission, the collected data is transferred to more powerful processing units such as computers, from which monitoring or querying is provided.

Smart classroom project [11] was conducted to help students. Information is collected from various sensor nodes that are responsible for providing different data. Once, the information reaches the central server, a complex event processing rule engine outputs some useful information. CEP system has recently emerged as a prominent technology to process real-time sensor data and create online responses to events [12].

In this study, a complex event processing based software framework for smart spaces is designed. The main aim of this study is that (1) designing low cost sensor nodes based on Arduino, (2) implementing these nodes in home environments to collect various information and then processing this sensor information at runtime to deduce useful information. In this context, data is collected by using many different types and number of sensors, which are Internet of Thing (IoT) 
devices, and then this data is sent to a central server via a gateway for event processing based on predefined rules. In the framework design, it is aimed not only to notify the mobile users via smartphones but to allow the users to change the rules by means of accessing rule sets of the framework as well.

\section{DETAILED ANALYSIS OF THREE SMART HOME SYSTEMS}

In order to focus on reasonable requirements, we have to know technological development in the related field. To do so, we have analyzed three known smart home studies in terms of hardware, software, method and communication technology in Table 3. Gator [8], MyHome [13], DOMOSEC [14] are smart home studies analyzed.

Table 3. Analyse of Three Smart Home System

\begin{tabular}{|c|c|c|c|}
\hline Criteria/Applications & $\begin{array}{l}\text { The Gator Tech Smart } \\
\text { Home }\end{array}$ & $\begin{array}{l}\text { OSGi-based } \\
\text { MyHome }\end{array}$ & DOMOSEC \\
\hline Hardware & 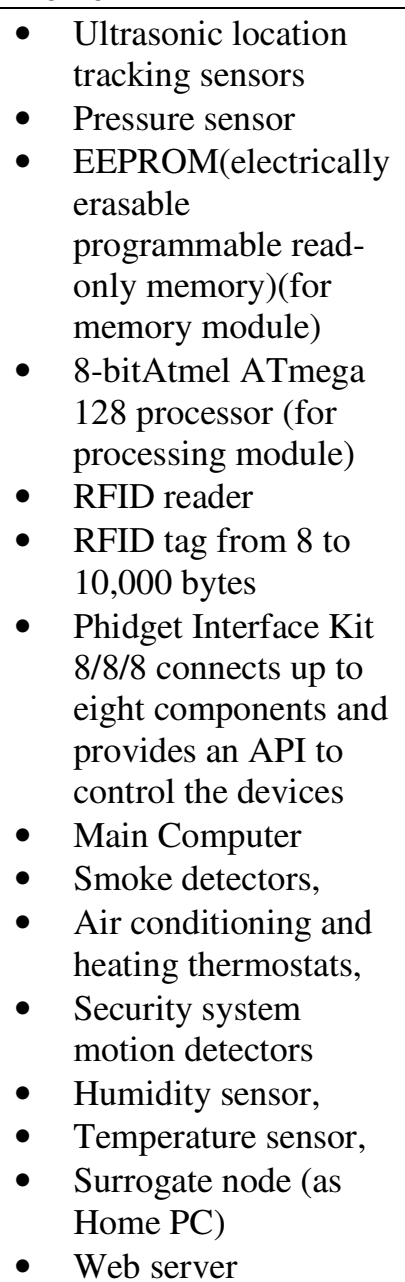 & $\begin{array}{ll} & \text { Health care } \\
\text { devices } \\
\text { - } & \text { GPS } \\
\text { - } & \text { Security sensors } \\
\text { - } & \text { Zigbee End } \\
\text { Devices(ZED) } \\
\text { - } \text { Zigbee } \\
\text { Coordinator } \\
\text { (ZC) } \\
\text { - } \text { Blood pressure } \\
\text { meter } \\
\text { - IP camera }\end{array}$ & $\begin{array}{ll}- & \text { Web-based } \\
& \text { gateway } \\
\text { - } & \text { A PC based } \\
& \text { gateway } \\
\text { - } & \text { Noise and door } \\
\text { opening } \\
\text { detectors } \\
\text { - X10 module } \\
\text { - } \quad \text { IP camera }\end{array}$ \\
\hline $\begin{array}{l}\text { Software/ Core } \\
\text { Technology }\end{array}$ & $\begin{array}{ll}\text { - } & \text { Sensor driver } \\
\text { - } & \text { Firmware for each } \\
& \text { sensor node } \\
\text { - } & \text { Open Services } \\
& \text { Gateway Initiative } \\
\end{array}$ & $\begin{array}{ll} & \text { Message } \\
\text { Oriented } \\
\text { Middleware } \\
\text { (MOM) } \\
\text { - } & \text { Open Service }\end{array}$ & $\begin{array}{ll}- & \text { Java } \\
\text { - } & \text { SCADA } \\
\text { (Supervisory } \\
\text { Control and } \\
\text { Data } \\
\end{array}$ \\
\hline
\end{tabular}


International Journal on Cloud Computing: Services and Architecture (IJCCSA) Vol. 7, No. 6, December 2017

\begin{tabular}{|c|c|c|c|}
\hline & (OSGi) framework & $\begin{array}{ll} & \text { Gateway } \\
& \text { Initiative } \\
& \text { (OSGi) } \\
& \text { framework } \\
\text { - } & \text { MySQL } \\
\text { database } \\
\text { - } & \text { PHP } \\
\text { - } & \text { Java script } \\
\text { - } & \text { MySQL } \\
& \text { database } \\
\text { - } & \text { Google Map } \\
& \text { API } \\
\text { - Java } \\
\text { Programming } \\
\text { Language and } \\
\text { Libraries } \\
\text { - ActiveMQ } \\
\text { Cross-platform } \\
\text { (inter-operate } \\
\text { on multiple } \\
\text { computer } \\
\text { platforms) } \\
\text { JVM } \\
\end{array}$ & $\begin{array}{l}\text { Acquisition) } \\
\text { software }\end{array}$ \\
\hline Method & $\begin{array}{ll}\text { - } & \text { Accurate location } \\
& \text { calculation, } \\
\text { - } & \text { Context builder } \\
\text { - } & \text { Context engine } \\
\text { - } & \text { Reasoning engine } \\
\text { - } & \text { Service composer } \\
\text { - X10 protocol }\end{array}$ & $\begin{array}{ll}\text { - } & \text { Service } \\
\text { Oriented } \\
\text { Architecture } \\
\text { - } & \text { ActiveMQ }\end{array}$ & $\begin{array}{l}\text { UPnP (Universal } \\
\text { Plug and Play) } \\
\text { The OSGi } \\
\text { framework } \\
\text { - The SHAP } \\
\text { Protocol }\end{array}$ \\
\hline $\begin{array}{l}\text { Communication } \\
\text { Technology }\end{array}$ & $\begin{array}{ll}\text { - } & \text { RF wireless } \\
& \text { communication } \\
\text { - } & \text { 10BaseT Ethernet } \\
\text { module } \\
\text { - } & \text { Low-power Wi-Fi } \\
\text { - } & \text { Power line } \\
& \text { communication }\end{array}$ & $\begin{array}{ll} & \text { ZigBee wireless } \\
& \text { communication } \\
\text { - } & \text { E-mail } \\
\text { - } & \text { Short message } \\
\text { - } & \text { RFID } \\
& \text { technology } \\
\text { - } & \text { RS232 }\end{array}$ & $\begin{array}{ll}\text { - } & \text { IP-based } \\
& \text { network } \\
\text { - } & \text { ZigBee } \\
\text { - } & \text { EIB (European } \\
& \text { Installation Bus) } \\
\text { - } & \text { Radio frequency } \\
& \text { (RF) } \\
\text { - } & \text { UDP protocol } \\
\text { - } & \text { Bluetooth } \\
\text { - } & \text { ADSL, ISDN or } \\
& \text { cable-modem } \\
\text { - } & \text { IP network } \\
- & \text { 6LoWPAN } \\
& \text { (IPv6 over Low } \\
& \text { power Wireless } \\
& \text { Personal Area } \\
& \text { Networks) } \\
\text { communications }\end{array}$ \\
\hline
\end{tabular}

Apart from the projects presented in Table 3, We have also investigated other smart home applications in this domain such as Aura [15], MavHome [16], Gaia [17] and MISS [18] projects 
in order to define state of the art. Also based on comparisons given in Table 3, we can draw out some state of the art solutions below.

\subsection{Software and core technology}

OSGi framework becomes a state of the art for connecting heterogeneous devices in indoor part. Some commercial and open-source OSGi framework implementations are Knopflerfish which is used by MyHome and MISS, Oscar, Apache Felix, Eclipse Equinox. In addition, King et al.[19] have developed a modular service oriented platform named as Atlas which enables each sensor and actuator node as a software service for smart spaces. This framework also allows to connect various heterogeneous devices easily. Web Services for outdoor applications should be used. The most important part is that smart home may include different network architectures such as Ethernet-based, Wi Fi, and ZigBee. In order to communicate these networks by different messaging type, the Message Oriented Middleware becomes a state of the art. Semantic-based context aware toolkit becomes state of the art. OSGi bundles are coded in java and added to the desired framework. Service composition and automatic service composition becomes state of the art. The previous applications used I/O matching service composition.

\subsection{Hardware technology}

Various type of sensors (/actuators) are used in smart home which are phidgets, telos motes, Insteon, X10 and various other types of intelligent sensors. However, they did not mention about model and properties of these sensors. Therefore, we could not compare this hardware. On the other hand, during the literature review, we can conclude that imote becomes new generation sensors. Some features of Telos sensors are that $250 \mathrm{kbps}$ data rate, 2.4 GHz IEEE 802.15.4 (Zigbee), Range: up to 50m indoors / 125m outdoors, Low Power, Fast Wakeup, Chipcon Wireless Transceiver $250 \mathrm{kbps}$ data rate. These become minimum requirements for sensors.

The recent smart home applications such as MyHome, Domosec have used IP-based camera instead of serial line for location detection by means of image processing. The web-based home gateway is used in most applications such as Amigo, Gator, Domosec, MyHome. In addition, X10 enabled devices also become state of the art. Touch screen based LCD screens becomes state of the art. RFID tags and readers are mostly used in recent applications and also become state of the art. Most applications start to use GPS-enabled devices for outdoor location detection.

\subsection{Communication protocol}

The previous applications have mostly used wired and limited wireless technology. However, depending on recent development on wireless technology, wireless communication becomes more popular. Ahmed et al. [20] have investigated three communication technologies which are Ethernet, WiFi and ZigBee for smart buildings. Wireless and Bluetooth architectures are used for already built houses. Wireless mesh network becomes state of the art due to robust and secure connectivity. ZigBee communication protocol based on low data rate for home devices such as washing machine, lamp, TV and Wi Fi protocols based on high data rate for multimedia devices become state of the art. Since energy consumption becomes primary issue in wireless devices, $\mathrm{ZigBee}$ is implemented in the recent home applications because of its low energy usage. Service discovery protocols such as Jini, UPnP, SLP becomes state of the art. The Aura did not use service discovery protocols. The lack of service discovery property makes their work more language-dependent and platform-dependent. 


\section{MATERIAls AND METHODS}

Arduino uno and mega boards are used to collect the sensor information through various sensors connected to the board. IoT devices based on Arduino for creating smart spaces have recently used in many applications. Ome and Rao [21] have implemented a low cost smart space application by using Arduino Due and ESP8266 wifi card to send collected sensor information to the Thing Speak cloud platform. In this paper, to design an event based system and inform users via mobile phone, a CEP framework is developed. The tool is implemented on the Android devices. The light, temperature, humidity, sound, rain, soil moisture and motion sensors are equipped to Arduino boards and the system starts to collect the sensor information which were later sent to a central server. The Arduino board is connected to an access point via an ESP8266 wifi module and then the collected data is sent to a central server with predefined labels via a gateway. The mobile users are registered to the desired event topics through publish-subscribe mechanism which were implemented on the central cloud server in Java. Whenever an event is occurred the result is sent to the subscribed mobile users. An alarm including event information popped up on the mobile users' smartphones.

In this part, definitions are also provided. Event terms is used as state change in application domain. Whenever an event is happened, which is already a record of some state change and this is immutable that means it is not possible to change the fired event. This constraint is a prominent definition for development of various systems.

Service Oriented Architectures (SOA): it is designed on top of three main parts these are service discovery, service registry and service provider mechanisms. A service registry is used by service provider to register its services. By doing this the service provider publishes its services. A service registry also presents these services to clients which send search queries to desired services. These services can be provided to both users and other services which provide their services through some part of other services.

Event Driven Architecture (EDA): In an event-driven software architecture style, a prominent thing occurs inside or outside of a structure, which distributes instantly to all interested parties (human or automated). The clients which are interested with issues process the event, and optionally produce an action. The event-driven action may consist of the invocation of a service, the triggering of a process, and extra information presentation.

Service Discovery: To find predefined services through service descriptions which are published and represented using metadata.

Message Oriented Middleware (MOM): It is distributing messages between parts in ubiquitous computing systems by synchronously or asynchronously.

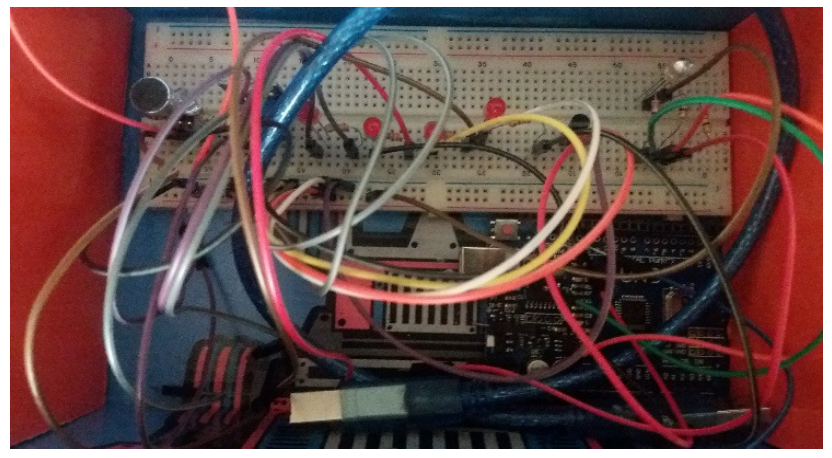

Figure 1. A prototype Arduino board and equipped sensors 
Designing various low-cost home sensor nodes has gained importance in the last decade. Chattoraj [22] has also designed an inexpensive smart home application based on Arduino to help the disabled and elderly. As seen in Figure 1, this is the instance of a sensor node designed to collect required data in this study. We have connected several sensors to Arduino board. Some of these sensors are;

- Light sensor

- Temperature and humidity sensor

- Sound sensor

- Motion sensor

- Rain/Snow sensor

- Moisture Sensor

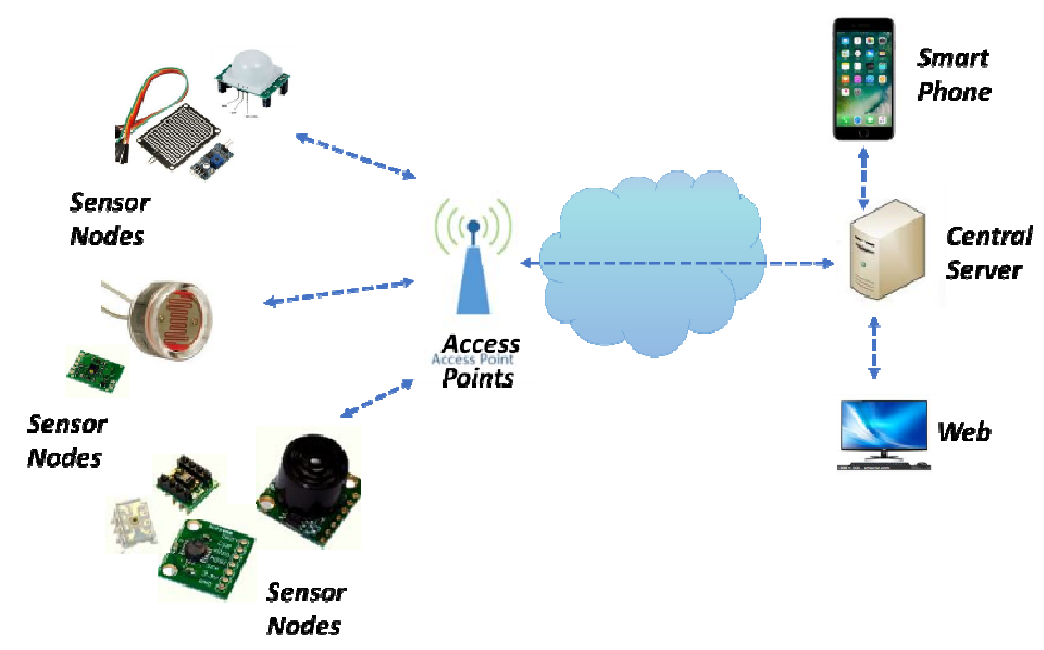

Figure 2. Framework architecture

In this study, sensor nodes are created and placed in the different locations. Figure 2 shows the main architecture of the framework. Sensors' data are sent to a central server through an access point. Sensor nodes use an ESP8266 wifi card for communication. Java socket is used to collect data on the server side. On the central server, the rules are defined by a rule engine. Drools rule engine is used in this study.



Figure 3. An example Drools rule format [23]

Figure 3 shows the rule format of drools rule engine [23]. As seen, facts and events are defined. 
In example, temperature data is collected from a sensor and an average of these data is calculated for last 10 minutes. If this calculated average value is over a threshold value, an alarm will be activated. If an event occurs, the drools rule engine sends required notification to an event based bus with a predefined topic. The mobile users who subscribed the predefined topics will be notified in case of any event.

\section{RESULTS AND DISCUSSION}

Sensor information is sent to a central server where meaningful information is extracted through a rule engine. A general publish-subscribe based event system was developed for various systems. For example, these systems are used in greenhouses, factories, homes and in various environments.

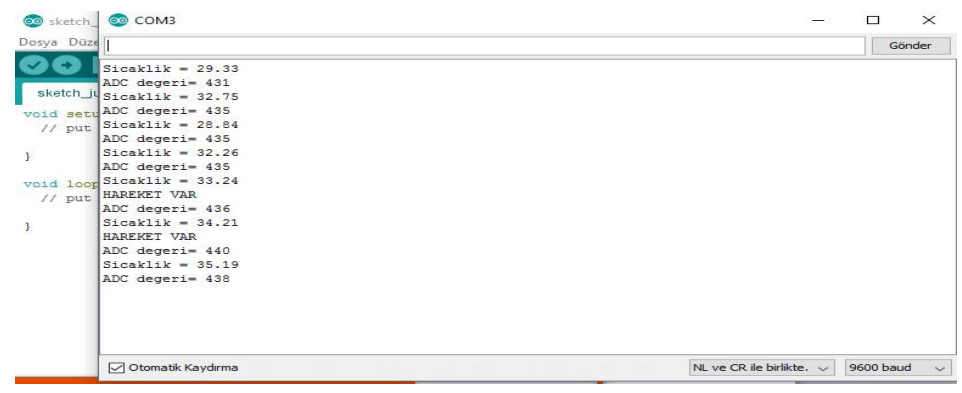

Figure 4. Sensor Information instance

Figure 4 shows a sample information collected from several Arduino uno sensor nodes. This information is sent to a central server in predefined intervals. An example scenario is implemented to test the framework. In addition, Kyas [24] presents several example scenarios. In this scenario, the user wakes up 30 minutes earlier when there is raining or snowing. The aim of waking up at 6:30 instead of at 7:00 is to avoid missing the meeting due to possible traffic congestion. The actions that will be executed by the rule are given below.

- First, obtain the current weather condition from the sensor at 6:30.

- Second, obtain the value(on/off) of Alarm.

- Third, identify whether the weather sensor value includes "rain" or "snow" text string.

- Forth, if the text string contains "rain" or "snow" and Alarm includes "on", starts alarm.

- Last, sets Alarm to "off". 


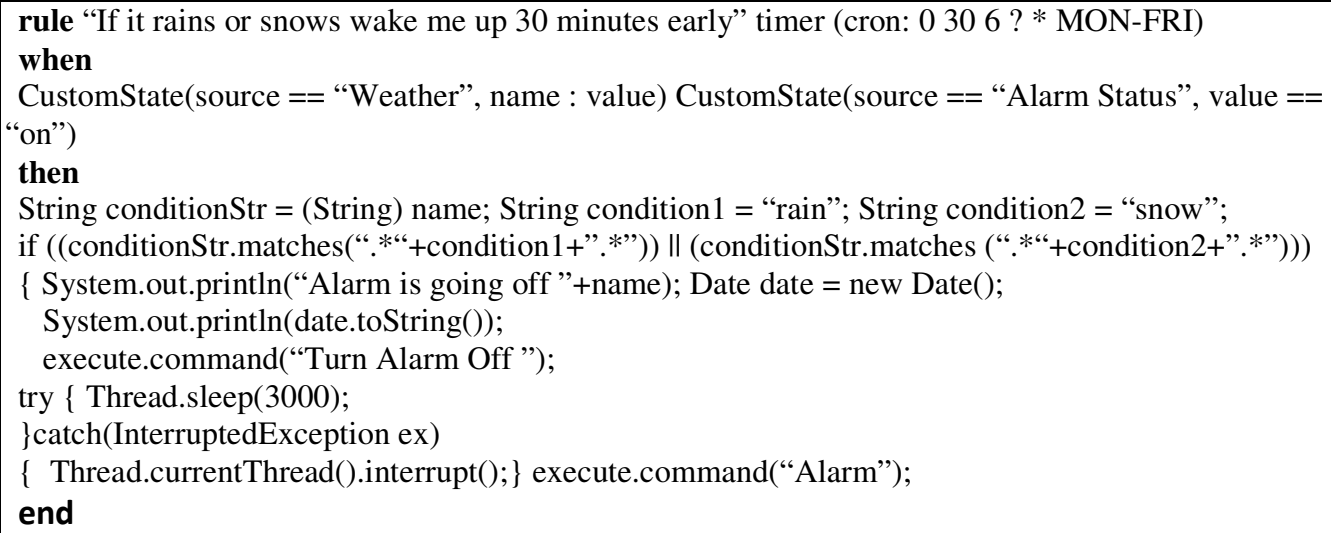

In this study, several sensors are used to test the framework; however, the range of sensor type could be extended in the future study. ESP8266 wifi module is used for communication, but even if this module is so cheap, the wifi module integrated to the board may be more functional and less error prone. In addition to this, it may also be easier to prepare a sensor node. The communication can also be achieved by using a cellular communication card. Besides this, the functionality of the framework can be increased by doing rule injection to drools at runtime.

\section{CONCLUSION}

In this study, several sensors are equipped to an Arduino uno board and a sensor node is created to collect necessary information. Furthermore, to make life easier and help people, the event based smart home application is implemented. Using various sensors type to collect desired information and then making meaningful information not only to help people for decision-making but also to protect them from several undesired situations or warn them.

Sensor nodes including an Arduino uno board and various sensors such as light, temperature, humidity, sound, rain and snow, soil moisture are used in this study to create a smart space. A complex event processing system is designed to notify the mobile users who is subscribed to predefined event topics if an event occurs. On the other hand, Although ESP8266 wifi module is cheap and useful to be used in this case, to use a wifi module integrated to Arduino board may be easier to prepare a sensor node. For further study, we will design a mobile rule injection interface to Drools rule engine at runtime.

\section{REFERENCES}

[1] Robles, Rosslin John, and Tai-hoon Kim. "Applications, Systems and Methods in Smart Home Technology: A." Int. Journal of Advanced Science And Technology 15 (2010).

[2] Cook, Diane J., and Sajal K. Das. "How smart are our environments? An updated look at the state of the art." Pervasive and mobile computing 3, no. 2 (2007): 53-73.

[3] Perera, Charith, Arkady Zaslavsky, Peter Christen, and Dimitrios Georgakopoulos. "Context aware computing for the internet of things: A survey." IEEE Communications Surveys \& Tutorials 16, no. 1 (2014): 414-454.

[4] Gubbi, Jayavardhana, Rajkumar Buyya, Slaven Marusic, and Marimuthu Palaniswami. "Internet of Things (IoT): A vision, architectural elements, and future directions." Future generation computer systems 29, no. 7 (2013): 1645-1660.

[5] Zoumboulakis, Michael, and George Roussos. "Escalation: Complex event detection in wireless sensor networks." Smart Sensing and Context (2007): 270-285.

[6] Yao, Wen, Chao-Hsien Chu, and Zang Li. "Leveraging complex event processing for smart hospitals using RFID." Journal of Network and Computer Applications 34, no. 3 (2011): 799-810. 
[7] Kidd, Cory, Robert Orr, Gregory Abowd, Christopher Atkeson, Irfan Essa, Blair MacIntyre, Elizabeth Mynatt, Thad Starner, and Wendy Newstetter. "The aware home: A living laboratory for ubiquitous computing research." Cooperative buildings. Integrating information, organizations, and architecture (1999): 191-198.

[8] Helal, Sumi, William Mann, Hicham El-Zabadani, Jeffrey King, Youssef Kaddoura, and Erwin Jansen. "The gator tech smart house: A programmable pervasive space." Computer 38, no. 3 (2005): 50-60.

[9] Hussain, Sajid, Scott Schaffner, and Dyllon Moseychuck. "Applications of wireless sensor networks and RFID in a smart home environment." In Communication Networks and Services Research Conference, 2009. CNSR'09. Seventh Annual, pp. 153-157. IEEE, 2009.

[10] Killeen, Peter, John Monkus, Biz Klessig, D. Hearn, Jingxian Wu, and Scott C. Smith. "Developing a Smart Home System." ESA'11 (2011): 1-3.

[11] Özlü, Fatih, Bilgin Avenoğlu, and P. Erhan Eren. "A complex event processing based framework implementation for ambient intelligence." In Signal Processing and Communications Applications Conference (SIU), 2013 21st, pp. 1-4. IEEE, 2013.

[12] Pooja, K. S., K. T. Chandrashekar, M. Thungamani, and C. N. Gireesh Babu. "Complex Event Processing In Smart Homes." management 4 (2015): 8.

[13] Huang, Hsuan-Yu, Wei-Chung Teng, and Sheng-Luen Chung. "Smart home at a finger tip: OSGi-based MyHome." In Systems, Man and Cybernetics, 2009. SMC 2009. IEEE International Conference on, pp. 4467-4472. IEEE, 2009.

[14] Zamora-Izquierdo, Miguel A., José Santa, and Antonio F. Gómez-Skarmeta. "An integral and networked home automation solution for indoor ambient intelligence." IEEE Pervasive Computing 9 , no. 4 (2010): 66-77.

[15] Garlan, David, Daniel P. Siewiorek, Asim Smailagic, and Peter Steenkiste. "Project aura: Toward distraction-free pervasive computing." IEEE Pervasive computing 1, no. 2 (2002): 22-31.

[16] Cook, Diane J., Michael Youngblood, Edwin O. Heierman, Karthik Gopalratnam, Sira Rao, Andrey Litvin, and Farhan Khawaja. "MavHome: An agent-based smart home." In Pervasive Computing and Communications, 2003. (PerCom 2003). Proceedings of the First IEEE International Conference on, pp. 521-524. IEEE, 2003.

[17] Román, Manuel, Christopher Hess, Renato Cerqueira, Anand Ranganathan, Roy H. Campbell, and Klara Nahrstedt. "A middleware infrastructure for active spaces." IEEE pervasive computing 1, no. 4 (2002): 74-83.

[18] Álamo, José M. Reyes, Hen-I. Yang, Johnny Wong, and Carl K. Chang. "Automatic service composition with heterogeneous service-oriented architectures." In International Conference on Smart Homes and Health Telematics, pp. 9-16. Springer, Berlin, Heidelberg, 2010.

[19] King, Jeffrey, Raja Bose, Hen-I. Yang, Steven Pickles, and Abdelsalam Helal. "Atlas: A serviceoriented sensor platform: Hardware and middleware to enable programmable pervasive spaces." In local computer networks, proceedings 2006 31st IEEE conference on, pp. 630-638. IEEE, 2006.

[20] Ahmed, Mohamed A., Yong Cheol Kang, and Young-Chon Kim. "Communication network architectures for smart-house with renewable energy resources." Energies 8, no. 8 (2015): 8716-8735.

[21] Ome, Nerella and Someswara Rao "Internet of Things (IoT) based Sensors to Cloud system using ESP8266 and Arduino Due", International Journal of Advanced Research in Computer and Communication Engineering, Vol. 5, Issue 10, 2016.

[22] Chattoraj, Subhankar. "Smart Home Automation based on different sensors and Arduino as the master controller." International Journal of Scientific and Research Publications 5, no. 10 (2015): 1-4.

[23] Anonymous, Drools JBoss, https://docs.jboss.org/drools/release/6.2.0.CR3/droolsdocs/html/DroolsComplexEventProcessingChapter.html. (Access date: 1.10.2017).

[24] Kyas, Othmar. How To Smart Home: A Step by Step Guide to Your Personal Internet of Things. Key Concept Press, 2015.

\section{Authors}

Dr. Mahir Kaya is an assistant professor at department of Computer Engineering at Gaziosmanpaşa University, Tokat, Turkey. His research interests focus on mobile cloud computing, internet of thing, code offloading, machine learning, cloud computing.

Dr. Yasemin Çetin-Kaya is an assistant professor at department of Computer Engineering at Gaziosmanpaşa University, Tokat, Turkey. Her research interests fall in machine learning, deep learning and Neural Network. 
International Journal on Cloud Computing: Services and Architecture (IJCCSA) Vol. 7, No. 6, December 2017 